\title{
36. PHYSICAL PROPERTIES OF LEG 65 BASALTS ${ }^{1}$
}

\author{
N. I. Christensen, Geological Sciences Department and Geophysics Program, \\ University of Washington, Seattle, Washington \\ and \\ B. T. R. Lewis, Department of Oceanography and Geophysics Program, \\ University of Washington, Seattle, Washington
}

\begin{abstract}
Velocities and densities of submarine basalts obtained during Leg 65 are distinguished by their relatively high values (about $6.3 \mathrm{~km} / \mathrm{s}$ and $2.9 \mathrm{~g} / \mathrm{cm}^{3}$, respectively). This is consistent with their low degree of alteration. The range of velocities covered by these samples correlates well with porosity, and a comparison with in situ velocities from refraction data suggests maximum porosities in the upper 100 meters of the crust of about $10 \%$.
\end{abstract}

\section{INTRODUCTION}

One of the major objectives of the Deep Sea Drilling Project (DSDP) during the International Phase of Ocean Drilling has been to drill a series of holes up to several kilometers deep into the oceanic crust. In the $\mathrm{Pa}$ cific these attempts have been generally abortive, in part because of the nature of the basalts near the surface of the crust. Prior to Leg 65 the other major efforts at deep crustal drilling in the Pacific were on Leg 54 at about $9^{\circ} \mathrm{N}$ and on Leg 34 on the Nazca Plate. Drilling here achieved penetrations of only a few tens of meters. A postmortem of these experiences suggested that a thin sediment cover (less than $100 \mathrm{~m}$ ) and the presence of glassy pillow basalts with a large fraction of water-filled voids between the pillows was responsible for the poor drilling conditions. It seems reasonable that such material would have a very low compressional wave velocity (of the order of $3.5 \mathrm{~km} / \mathrm{s}$ ) because of the high porosity.

Because of these êxperiences, one criterion for site selection after Leg 54 was that the uppermost crust have a reasonably high velocity-of the order of $4.5 \mathrm{~km} / \mathrm{s}$ or higher. It was thought that this would indicate basaltic rocks with a low porosity, either because the cracks and voids common in such rocks were filled with secondary minerals or because the ratio of pillow basalts to more massive basalt flows was small. Thus, in selecting sites at the mouth of the Gulf of California, the criterion was that there be at least 100 meters of sediment overlying igneous crust with a velocity of about $4.5 \mathrm{~km} / \mathrm{s}$ or greater.

Site survey results using OBS refraction data suggested that such sites existed in sediment-filled valleys a few kilometers south of the Tamayo Fracture Zone. A velocity-depth profile for the crust under Sites 482 and 485 from McClain and Lewis (1980) is shown in Figure 1 . These results suggest that the desired criterion was present at these sites.

\footnotetext{
${ }^{1}$ Lewis, B. T. R., Robinson, P., et al., Init. Repts. DSDP, 65: Washington (U.S. Govt.
}

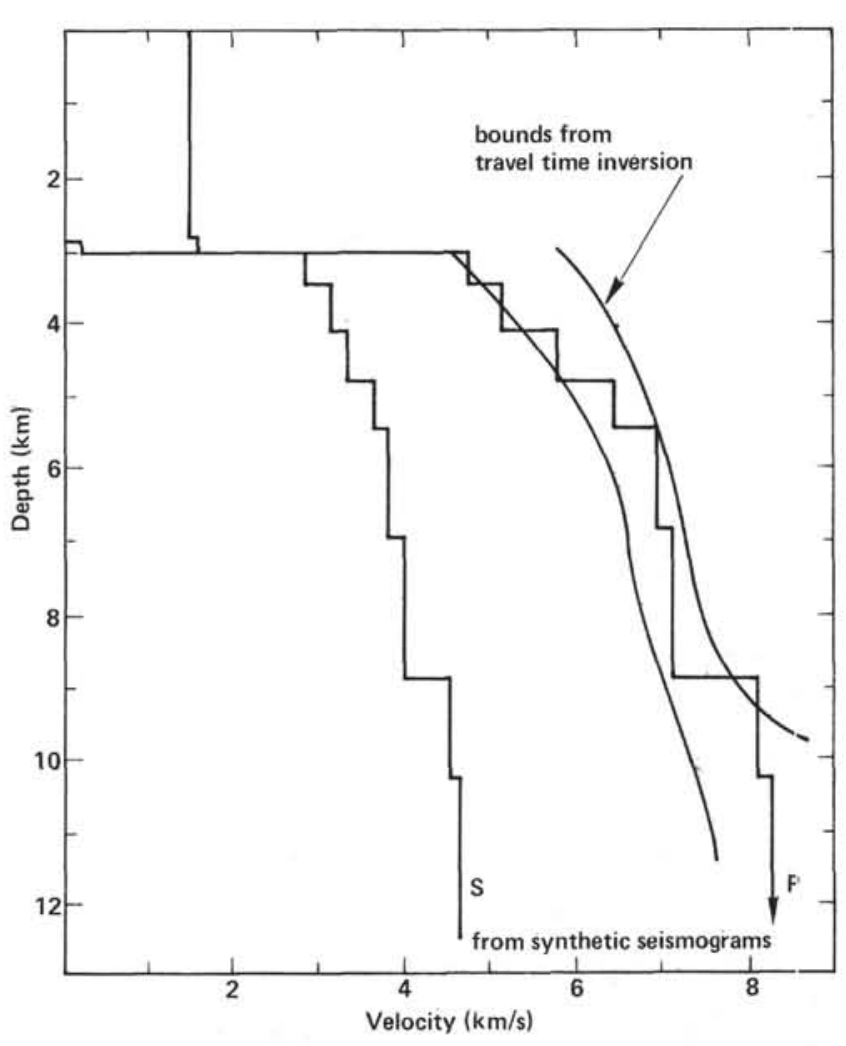

Figure 1. Velocity model applicable to Sites 482 and 485 from interpretation of refraction data (McClain and Lewis, 1980).

The drilling conducted on Leg 65 revealed that the relatively high velocities are probably related to the presence of massive basalt flows interlayered with sediments and to the general absence of pillow basalts. Lewis (1980) has suggested that these basalt flows were ponded in pre-existing axial valleys at the ridge crest and that, because of these conditions and the high sedimentation rate at the mouth of the Gulf of California, this area may not be typical of portions of the East Pacific Rise displaying axially high topography. Clearly a study 
of the velocities in basalts from this site is significant in the interpretation of seismic structure.

In this chapter we present laboratory measurements of the physical properties of basalts drilled on Leg 65 and compare the measurements of compressional wave velocities with the results from the refraction experiment.

\section{EXPERIMENTAL TECHNIQUES AND DATA}

Compressional $\left(V_{p}\right)$ and shear wave $\left(V_{s}\right)$ velocities are presented in Table 1 with wet-bulk densities $(\varrho)$, porosities $(\phi)$, ratios of compressional to shear velocities $\left(V_{p} / V_{s}\right)$, Poisson's ratios $(\sigma)$, bulk moduli $(K)$, shear moduli $(\mu)$, Lamés' constants $(\lambda)$, and Young's moduli $(E)$.

The samples selected for the velocity and density measurements were cut into cores $2.5 \mathrm{~cm}$ in diameter and 3 to $5 \mathrm{~cm}$ in length. The densities were calculated from the core volumes and their watersaturated weights. The porosities are "effective porosities" calculated from wet and dry weights.

The pulse transmission technique described in detail by Birch (1960) was used to obtain the compressional and shear wave velocities through the cores. Barium titanate and AC-cut quartz transducers of 1 to $2 \mathrm{MHz}$ frequencies were used to generate and receive compressional and shear waves, respectively. Pore pressures were maintained at values lower than external pressures by placing 100 mesh screen between the samples and the copper jackets used to exclude the pressure medium. The transducers were coupled to the samples with rubber tubing and pressure applied in 0.2 and $1.0 \mathrm{kbar}$ steps. The pressure-generating system contains a two-stage intensifier using a low-viscosity oil for the pressure medium. Pressure was measured with a calibrated manganin coil located on the high pressure side of the intensifier.

The velocities are estimated to be accurate to within 1\% (Christensen and Shaw, 1970). Elastic constants were calculated from the measured velocities and densities using the equations summarized by Birch (1961). The velocities and densities used for the calculations and given in Table 1 were corrected for compression, using an iterative technique and the dynamically determined compressibilities.

\section{DISCUSSION}

One of the distinguishing features of the basalts from Leg 65 is their relatively high velocities (Table 1). Previous studies have found that compressional and shear wave velocities in DSDP basalts at elevated confining pressures show extreme variability and can be as low as 3.5 and $1.7 \mathrm{~km} / \mathrm{s}$, respectively (Christensen and Salisbury, 1975). The low velocities are accompanied by relatively low densities (often less than $2.5 \mathrm{~g} / \mathrm{cm}^{3}$ ) and, in general, result from low temperature alteration. The Leg 65 basalts, on the other hand, are extremely fresh, with densities generally above $2.9 \mathrm{~g} / \mathrm{cm}^{3}$.

Petrographic examination of thin sections cut from the samples in which velocities were measured show relatively little alteration. Estimated modal analyses are given in Table 2 . The basalts can be subdivided texturally into equigranular aphyric and porphyritic types. Both contain abundant calcic plagioclase and augite. The plagioclase in the aphyric basalts is commonly intergrown with augite in an ophitic texture. The porphyritic rocks contain plagioclase phenocrysts enclosed in a microcrystalline groundmass composed of augite, plagioclase, and opaque minerals. The absence of flow fabrics in both textural types suggests crystallization under stagnant conditions and, in turn, results in isotropic elastic properties. This supports the ponding model for these basalts proposed by Lewis (1980).

In Figure 2, compressional wave velocities at $1 \mathrm{kbar}$ confining pressure are plotted as a function of density.
Table 1. Velocities, densities, porosities, and elastic constants of Leg 65 basalts.

\begin{tabular}{|c|c|c|c|c|c|c|c|c|}
\hline $\begin{array}{c}\text { Sample } \\
\text { (interval in } \mathrm{cm} \text { ) }\end{array}$ & $\begin{array}{l}\text { Confining } \\
\text { Pressure } \\
\text { (kbar) }\end{array}$ & $\underset{(\mathrm{km} / \mathrm{s})}{V_{p}}$ & $\begin{array}{l}V_{V_{5}} \\
(\mathrm{~km} / \mathrm{s})\end{array}$ & $V_{p} / v_{s}$ & $\sigma$ & $\stackrel{{ }_{(M)}^{K}}{ }$ & (Mb) & $\stackrel{\stackrel{\lambda}{(M b)}}{ }$ \\
\hline $482 \mathrm{~B}-16-2,107-123$ & 0.2 & 6.27 & 3.42 & 1.83 & 0.29 & 0.70 & 0.35 & 0.47 \\
\hline & 0.4 & 6.29 & 3.44 & 1.83 & 0.29 & 0.70 & 0.35 & 0.47 \\
\hline & 0.6 & 6.30 & 3.45 & 1.83 & 0.29 & 0.70 & 0.35 & 0.47 \\
\hline & 0.8 & $\begin{array}{l}6.31 \\
6,32\end{array}$ & $\begin{array}{l}3.45 \\
3.46\end{array}$ & 1.83 & 0.29 & 0.71 & 0.35 & 0.47 \\
\hline & 2.0 & $\begin{array}{l}6.32 \\
6.35\end{array}$ & $\begin{array}{l}3.46 \\
3.47\end{array}$ & $\begin{array}{l}1.83 \\
1.83\end{array}$ & $\begin{array}{l}0.29 \\
0.29\end{array}$ & 0.72 & $\begin{array}{l}0.35 \\
0.36\end{array}$ & $\begin{array}{l}0.47 \\
0.48\end{array}$ \\
\hline & 4.0 & 6.42 & 3.48 & 1.84 & 0.29 & 0.74 & 0.36 & 0.50 \\
\hline & 6.0 & 6.48 & 3.49 & 1.86 & 0.30 & 0.76 & 0.36 & 0.52 \\
\hline $482 \mathrm{C}-10-3,73-85$ & 0.2 & 6.02 & 3.23 & 1.86 & 0.30 & 0.65 & 0.30 & 0.45 \\
\hline & 0.4 & 6.04 & 3.24 & 1.86 & 0.30 & 0.65 & 0.30 & 0.45 \\
\hline & $\begin{array}{l}0.6 \\
0.8\end{array}$ & $\begin{array}{l}6.06 \\
6.08\end{array}$ & $\begin{array}{l}3.26 \\
3.27\end{array}$ & $\begin{array}{l}1.86 \\
1.86\end{array}$ & ${ }_{30}^{30}$ & $\begin{array}{l}0.65 \\
0.66\end{array}$ & 31 & $\begin{array}{l}0.45 \\
0.45\end{array}$ \\
\hline & $\begin{array}{l}.8 \\
1.0\end{array}$ & $\begin{array}{l}6.08 \\
6.09\end{array}$ & 3.28 & $\begin{array}{l}1.80 \\
1.86\end{array}$ & $\begin{array}{l}0.30 \\
0.30\end{array}$ & $\begin{array}{l}0.00 \\
0.66\end{array}$ & 0.31 & 0.45 \\
\hline & 2.0 & 6.14 & 3.30 & 1.86 & 0.30 & 0.67 & 0.32 & 0.46 \\
\hline & 4.0 & 6.23 & 3.32 & 1.88 & 0.30 & 0.70 & 0.32 & 0.49 \\
\hline & 6.0 & 6.31 & 3.33 & 1.89 & 0.31 & 0.73 & 0.32 & 0.51 \\
\hline $482 \mathrm{C}-13-2,117-128$ & 0.2 & 6.23 & 3.38 & 1.84 & 0.29 & 0.69 & 0.34 & 0.47 \\
\hline $\begin{array}{l}\ell=2.94 \\
\phi=1.10\end{array}$ & 0.4 & $\begin{array}{l}6.25 \\
6.28\end{array}$ & $\begin{array}{l}3.40 \\
3.41\end{array}$ & 1.84 & 0.29 & $\begin{array}{l}0.70 \\
0.70\end{array}$ & 0.34 & 0.47 \\
\hline & $\begin{array}{l}0.6 \\
0.8\end{array}$ & $\begin{array}{l}6.30 \\
6.30\end{array}$ & $\begin{array}{l}3.41 \\
3.42\end{array}$ & $\begin{array}{l}1.84 \\
1.84\end{array}$ & $\begin{array}{l}0.29 \\
0.29\end{array}$ & $\begin{array}{l}0.70 \\
0.71\end{array}$ & $\begin{array}{l}0.34 \\
0.34\end{array}$ & $\begin{array}{l}0.48 \\
0.48\end{array}$ \\
\hline & 1.0 & 6.31 & 3.42 & 1.85 & 0.29 & 0.71 & 0.34 & 0.48 \\
\hline & 2.0 & 6.37 & 3.44 & 1.85 & 0.29 & 0.73 & 0.35 & 0.50 \\
\hline & 4.0 & 6.45 & 3.46 & 1.86 & & 0.76 & & 0.52 \\
\hline & $\begin{array}{l}6.0 \\
0.2\end{array}$ & $\begin{array}{l}6.50 \\
627\end{array}$ & $\begin{array}{l}3.47 \\
3.44\end{array}$ & 1.87 & & 0.77 & & \\
\hline 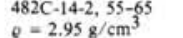 & $\begin{array}{l}0.2 \\
0.4\end{array}$ & $\begin{array}{l}6.27 \\
6.28\end{array}$ & $\begin{array}{l}3.44 \\
3.45\end{array}$ & $\begin{array}{l}1.82 \\
1.82\end{array}$ & $\begin{array}{l}0.28 \\
0.28\end{array}$ & $\begin{array}{l}0.69 \\
0.70\end{array}$ & $\begin{array}{l}0.35 \\
0.35\end{array}$ & $\begin{array}{l}0.46 \\
0.46\end{array}$ \\
\hline $0=0.90$ & 0.6 & 6.30 & $\begin{array}{l}3.43 \\
3.46\end{array}$ & 1.82 & 0.28 & 0.70 & 0.35 & 0.46 \\
\hline & 0.8 & 6.31 & 3.47 & 1.82 & 0.28 & 0.70 & 0.36 & 0.46 \\
\hline & 1.0 & 6.32 & 3.48 & 1.82 & 0.28 & 0.70 & 0.36 & 0.46 \\
\hline & 2.0 & 6.35 & 3.50 & 1.81 & & 0.71 & .36 & 0.47 \\
\hline & 4.0 & 6.42 & & 1.83 & & 0.73 & 0.36 & 0.49 \\
\hline $482 \mathrm{D}-9 \cdot 1,69-81$ & $\begin{array}{l}6.0 \\
0.2\end{array}$ & $\begin{array}{l}6.47 \\
6.31\end{array}$ & $\begin{array}{l}3.52 \\
3.43\end{array}$ & $\begin{array}{l}1.84 \\
1.84\end{array}$ & $\begin{array}{l}0.29 \\
0.29\end{array}$ & $\begin{array}{l}0.75 \\
0.71\end{array}$ & $\begin{array}{l}0.37 \\
0.35\end{array}$ & $\begin{array}{l}0.51 \\
0.48\end{array}$ \\
\hline & 0.4 & $\begin{array}{l}6.31 \\
6.36\end{array}$ & $\begin{array}{l}3.43 \\
3.45\end{array}$ & $\begin{array}{l}1.84 \\
1.84\end{array}$ & $\begin{array}{l}0.29 \\
0.29\end{array}$ & $\begin{array}{l}0.71 \\
0.73\end{array}$ & .35 & $\begin{array}{l}0.48 \\
0.49\end{array}$ \\
\hline$\phi=1.0$ & 0.6 & 6.39 & 3.46 & 1.85 & 0.29 & 0.73 & 0.35 & 0.50 \\
\hline & 0.8 & 6.41 & 3.47 & 1.85 & 0.29 & 0.74 & 36 & \\
\hline & 1.0 & 6.43 & & 1.85 & & & & \\
\hline & 2.0 & 6.47 & & 1.86 & & & & \\
\hline & $\begin{array}{l}4.0 \\
6.0\end{array}$ & $\begin{array}{l}6.51 \\
6.54\end{array}$ & $\begin{array}{l}3.49 \\
3.49\end{array}$ & $\begin{array}{l}1.87 \\
1.87\end{array}$ & 0.30 & 0.77 & 0.36 & 0.53 \\
\hline $482 \mathrm{D}-11-3,0-8$ & 0.2 & 6.36 & 3.35 & $\begin{array}{l}1.87 \\
1.90\end{array}$ & $\begin{array}{l}0.30 \\
0.31\end{array}$ & $\begin{array}{l}0.78 \\
0.75\end{array}$ & $\begin{array}{l}0.36 \\
0.33\end{array}$ & $\begin{array}{l}0.54 \\
0.53\end{array}$ \\
\hline$Q=2.94 \mathrm{~g} /$ & 0.4 & $\begin{array}{l}6.30 \\
6.38\end{array}$ & 3.38 & $\begin{array}{l}1.90 \\
1.89\end{array}$ & $\begin{array}{l}0.31 \\
0.30\end{array}$ & $\begin{array}{l}0.75 \\
0.75\end{array}$ & $\begin{array}{l}0.33 \\
0.34\end{array}$ & $\begin{array}{l}0.53 \\
0.53\end{array}$ \\
\hline$\delta=0.70$ & 0.6 & 6.39 & 3.40 & 1.88 & & 0.75 & 0.34 & \\
\hline & 0.8 & 6.40 & & 1.87 & & & & \\
\hline & 1.0 & 6.41 & 3.43 & 1.87 & & & & 0.52 \\
\hline & $\begin{array}{l}2.0 \\
4.0\end{array}$ & $\begin{array}{l}6.45 \\
6.50\end{array}$ & $\begin{array}{l}3.46 \\
3.50\end{array}$ & 1.86 & & 0.75 & 0.35 & 0.52 \\
\hline & $\begin{array}{l}4.0 \\
6.0\end{array}$ & 6.54 & 3.52 & $\begin{array}{l}1.86 \\
1.86\end{array}$ & $\begin{array}{l}0.30 \\
0.30\end{array}$ & $\begin{array}{l}0.76 \\
0.77\end{array}$ & $\begin{array}{l}0.36 \\
0.37\end{array}$ & $\begin{array}{l}0.52 \\
0.53\end{array}$ \\
\hline $483-15-2,129-140$ & 0.2 & 6.06 & 3.31 & 1.83 & 0.29 & 0.65 & $\begin{array}{l}0.37 \\
0.32\end{array}$ & $\begin{array}{l}0.33 \\
0.43\end{array}$ \\
\hline$e=2.93$ & 0.4 & 6.09 & 3.33 & 1.83 & 0.29 & 0.65 & 32 & \\
\hline$\delta=1,7 \%$ & 0.6 & 6.11 & & 1.83 & & & & \\
\hline & 0.8 & 6.12 & & 1.83 & & & & \\
\hline & $\begin{array}{l}1.0 \\
2.0\end{array}$ & $\begin{array}{l}6.14 \\
6.18\end{array}$ & $\begin{array}{l}3.36 \\
3.38\end{array}$ & $\begin{array}{l}1.83 \\
1.83\end{array}$ & & & & $\begin{array}{l}0.44 \\
0.45\end{array}$ \\
\hline & 4.0 & 6.25 & 3.41 & 1.83 & $\begin{array}{l}0.29 \\
0.29\end{array}$ & $\begin{array}{l}0.67 \\
0.69\end{array}$ & $\begin{array}{l}0.34 \\
0.34\end{array}$ & .46 \\
\hline & 6.0 & 6.30 & 3.44 & 1.83 & 0.29 & 0.70 & 0.35 & 0.47 \\
\hline $483-17-2,130-145$ & $\begin{array}{l}0.2 \\
0.4\end{array}$ & $\begin{array}{l}6.37 \\
638\end{array}$ & $\begin{array}{l}3.40 \\
3.42\end{array}$ & 1.87 & $\begin{array}{l}0.30 \\
0.30\end{array}$ & $\begin{array}{l}0.73 \\
0.73\end{array}$ & $\begin{array}{l}0.34 \\
0.34\end{array}$ & $\begin{array}{l}0.51 \\
0.51\end{array}$ \\
\hline$£$ & $\begin{array}{l}0.4 \\
0.6\end{array}$ & $\begin{array}{l}0.30 \\
6.40\end{array}$ & $\begin{array}{l}3.42 \\
3.44\end{array}$ & 1.86 & 0.30 & 0.74 & 0.35 & .51 \\
\hline & 0.8 & 6.41 & 3.45 & 1.86 & & & & \\
\hline & 1.0 & 6.42 & 3.46 & 1.86 & 30 & 0.74 & 0.35 & \\
\hline & 2.0 & 6.46 & & 1.86 & & & & \\
\hline & & 6. & & & & & & \\
\hline & 6.0 & & & & & & & \\
\hline$-1,69-8 !$ & 0.2 & 6. & & & & & & \\
\hline & $\begin{array}{l}0.4 \\
0.6\end{array}$ & $\begin{array}{l}6.35 \\
6.36\end{array}$ & $\begin{array}{l}3.40 \\
3.42\end{array}$ & $\begin{array}{l}1.87 \\
1.86\end{array}$ & & & & \\
\hline & 0.8 & & 3.43 & $\begin{array}{l}1.86 \\
1.86\end{array}$ & $\begin{array}{l}0.30 \\
0.30\end{array}$ & & 35 & \\
\hline & 1.0 & 6.38 & 3.44 & 1.85 & & & & \\
\hline & & & & & & & & \\
\hline & 4.0 & & & & & & & \\
\hline $483-22-3,7$ & 6.0 & & & 1.85 & & & & \\
\hline $\mathrm{Q}=2.93 \mathrm{~g}$ & 0.4 & & $\begin{array}{l}3.42 \\
3.44\end{array}$ & $\begin{array}{l}1.86 \\
1.86\end{array}$ & & & & \\
\hline 0 & 0.6 & & 3. & $\begin{array}{l}1.86 \\
1.86\end{array}$ & & & & \\
\hline & 0.8 & & & & & & & \\
\hline & 1.0 & & & & & & & \\
\hline & 2. & & & & & & & \\
\hline & $\begin{array}{l}4.0 \\
60\end{array}$ & & & 1.87 & & & & \\
\hline $483-24$ & $\begin{array}{l}6.0 \\
0.2\end{array}$ & & & & & & & \\
\hline & 0. & & & $\begin{array}{l}1.88 \\
1.83\end{array}$ & & & & \\
\hline & 0.6 & 5.8 & & & & & & \\
\hline & 0.8 & & & & & & & \\
\hline & $1.42-1$ & & & & & & & \\
\hline & & & & & & & & \\
\hline & 6. & & 3.30 & 1.8 & & & & \\
\hline $483-26-2,133-139$ & 0.2 & & 3. & 1.8 & & & & 0.43 \\
\hline & 0. & & & & & & & \\
\hline & 0. & 6.0 & 3.2. & 1. & & & & \\
\hline & 1. & & & & & & & \\
\hline & 2. & & & & & & & \\
\hline & 4 & & & & & & & \\
\hline & & & & & & & & \\
\hline & 0.2 & & & & & & & \\
\hline & 0.4 & & & 1.84 & & & & \\
\hline & 0.8 & & & $\begin{array}{l}1.84 \\
1.84\end{array}$ & & & & \\
\hline & 1. & & & & & & & \\
\hline & 2.0 & & & & & & & \\
\hline & 4.0 & 6.47 & $\begin{array}{l}3.51 \\
3.53\end{array}$ & 1.84 & 0.29 & & 0.36 & 0.51 \\
\hline & 6.0 & 6.52 & 3.53 & 1.85 & 0.29 & 0.76 & 0.37 & 0.51 \\
\hline
\end{tabular}


Table 1. (Continued).

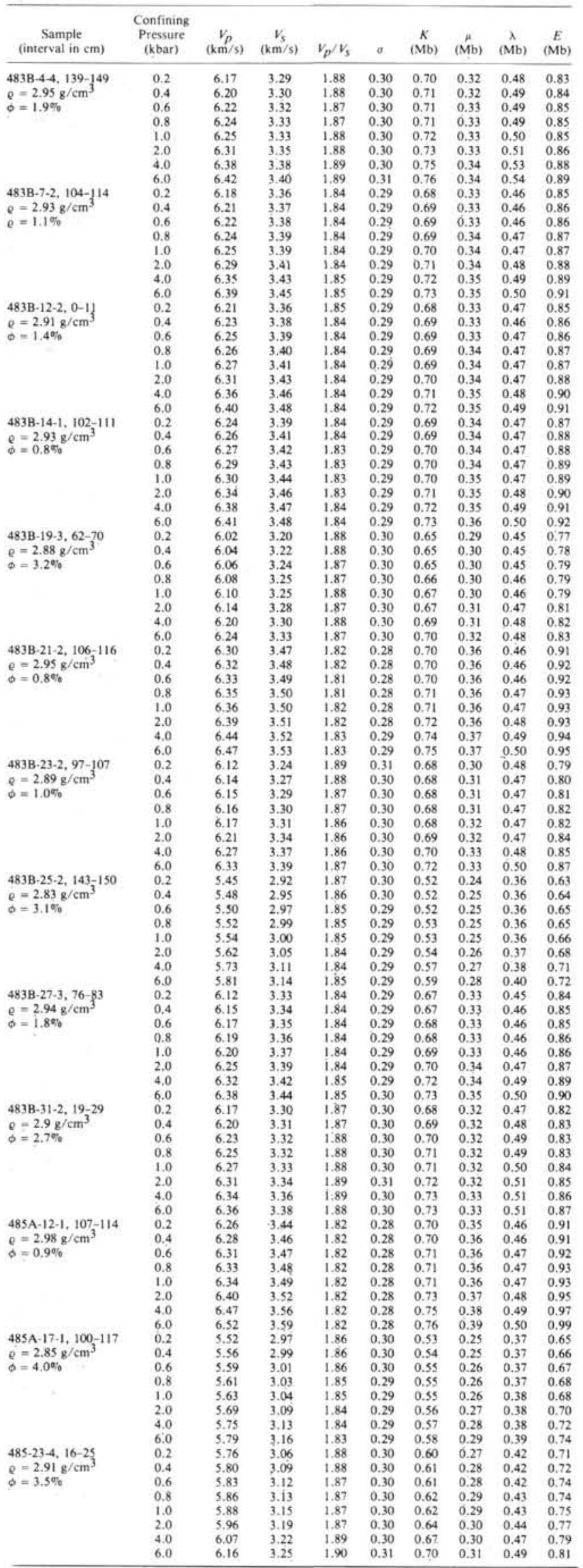

Table 1. (Continued).

\begin{tabular}{|c|c|c|c|c|c|c|c|c|c|}
\hline $\begin{array}{c}\text { Sample } \\
\text { (interval in } \mathrm{cm} \text { ) }\end{array}$ & $\begin{array}{c}\text { Confining } \\
\text { Pressure } \\
\text { (kbar) }\end{array}$ & $\begin{array}{c}V_{p} \\
(\mathrm{~km} / \mathrm{s})\end{array}$ & $\begin{array}{c}V_{s} \\
(\mathrm{~km} / \mathrm{s})\end{array}$ & $V_{p} / V_{s}$ & 0 & $\underset{(\mathrm{Mb})}{K}$ & $\stackrel{\mu}{\mu} \mathrm{Mb})$ & $\stackrel{\lambda}{(\mathrm{Mb})}$ & $\begin{array}{c}E \\
(\mathrm{Mb})\end{array}$ \\
\hline \multirow{8}{*}{$\begin{array}{l}\text { 485A-25-3.33-45 } \\
e=2.95 \mathrm{~g} / \mathrm{cm}^{3} \\
\phi=0.8 \%\end{array}$} & 0.2 & 6.08 & 3.31 & 1.84 & 0.29 & 0.66 & 0.32 & 0.44 & 0.83 \\
\hline & 0.4 & 6.11 & 3.32 & 1.84 & 0.29 & 0.67 & 0.33 & 0.45 & 0.84 \\
\hline & 0.6 & 6.14 & 3.33 & 1.84 & 0.29 & 0.68 & 0.33 & 0.46 & 0.85 \\
\hline & 0.8 & 6.16 & 3.34 & 1.84 & 0.29 & 0.68 & 0.33 & 0.46 & 0.85 \\
\hline & 1.0 & 6.17 & 3.35 & 1.84 & 0.29 & 0.68 & 0.33 & 0.46 & 0.86 \\
\hline & 2.0 & 6.22 & 3.37 & 1.85 & 0.29 & 0.70 & 0.34 & 0.47 & 0.87 \\
\hline & 4.0 & 6.30 & 3.40 & 1.85 & 0.29 & 0.72 & 0.34 & 0.49 & 0.88 \\
\hline & 6.0 & 6.37 & 3.42 & 1.86 & 0.30 & 0.74 & 0.35 & 0.51 & 0.90 \\
\hline \multirow{8}{*}{$\begin{array}{l}485 \mathrm{~A}-29-3,38-48 \\
e=2.94 \mathrm{~g} / \mathrm{cm}^{3} \\
\phi=1.0 \%\end{array}$} & 0.2 & 6.14 & 3.31 & 1.85 & 0.30 & 0.68 & 0.32 & 0.46 & 0.83 \\
\hline & 0.4 & 6.17 & 3.32 & 1.86 & 0.30 & 0.69 & 0.32 & 0.47 & 0.84 \\
\hline & 0.6 & 6.20 & 3.33 & 1.86 & 0.30 & 0.70 & 0.33 & 0.48 & 0.85 \\
\hline & 0.8 & 6.22 & 3.34 & 1.86 & 0.30 & 0.70 & 0.33 & 0.48 & 0.85 \\
\hline & 1.0 & 6.24 & 3.34 & 1.87 & 0.30 & 0.71 & 0.33 & 0.49 & 0.85 \\
\hline & 2.0 & 6.31 & 3.36 & 1.88 & 0.30 & 0.73 & 0.33 & 0.51 & 0.87 \\
\hline & 4.0 & 6.39 & 3.38 & 1.89 & 0.31 & 0.75 & 0.34 & 0.53 & 0.88 \\
\hline & 6.0 & 6.46 & 3.39 & 1.91 & 0.31 & 0.78 & 0.34 & 0.55 & 0.89 \\
\hline \multirow{8}{*}{$\begin{array}{l}\text { 485A-31-2, 94-101 } \\
e=2.92 \mathrm{~g} / \mathrm{cm}^{3} \\
\phi=1.8 \%\end{array}$} & 0.2 & 6.15 & 3.23 & 1.90 & 0.31 & 0.70 & 0.30 & 0.50 & 0.80 \\
\hline & 0.4 & 6.19 & 3.25 & 1.90 & 0.31 & 0.71 & 0.31 & 0.50 & 0.81 \\
\hline & 0.6 & 6.22 & 3.26 & 1.91 & 0.31 & 0.72 & 0.31 & 0.51 & 0.81 \\
\hline & 0.8 & 6.24 & 3.27 & 1.91 & 0.31 & 0.72 & 0.31 & 0.51 & 0.82 \\
\hline & 1.0 & 6.26 & 3.27 & 1.91 & 0.31 & 0.73 & 0.31 & 0.52 & 0.82 \\
\hline & 2.0 & 6.30 & 3.30 & 1.91 & 0.31 & 0.74 & 0.32 & 0.52 & 0.83 \\
\hline & 4.0 & 6.35 & 3.33 & 1.91 & 0.31 & 0.75 & 0.32 & 0.53 & 0.85 \\
\hline & 6.0 & 6.37 & 3.35 & 1.90 & 0.31 & 0.75 & 0.33 & 0.53 & 0.86 \\
\hline \multirow{8}{*}{$\begin{array}{l}485 \mathrm{~A}-33-1,32-42 \\
e=2.91 \mathrm{~g} / \mathrm{cm}^{3} \\
\delta=1.1 \%\end{array}$} & 0.2 & 6.26 & 3.24 & 1.93 & 0.32 & 0.73 & 0.31 & 0.53 & 0.80 \\
\hline & 0.4 & 6.30 & 3.28 & 1.92 & 0.31 & 0.74 & 0.31 & 0.53 & 0.82 \\
\hline & 0.6 & 6.33 & 3.30 & 1.92 & 0.31 & 0.74 & 0.32 & 0.53 & 0.83 \\
\hline & 0.8 & 6.36 & 3.32 & 1.92 & 0.31 & 0.75 & 0.32 & 0.54 & 0.84 \\
\hline & 1.0 & 6.38 & 3.33 & 1.92 & 0.31 & 0.75 & 0.32 & 0.54 & 0.85 \\
\hline & 2.0 & 6.48 & 3.37 & 1.92 & 0.31 & 0.78 & 0.33 & 0.56 & 0.87 \\
\hline & 4.0 & 6.53 & 3.41 & 1.91 & 0.31 & 0.79 & 0.34 & 0.57 & 0.89 \\
\hline & 6.0 & 6.59 & 3.45 & 1.91 & 0.31 & 0.80 & 0.35 & 0.57 & 0.91 \\
\hline \multirow{8}{*}{$\begin{array}{l}485 \mathrm{~A}-35-1 \\
\mathrm{e}=2.94 \mathrm{~g} / \mathrm{cm}^{3} \\
\oint=1.8 \%\end{array}$} & 0.2 & 6.09 & 3.32 & 1.83 & 0.29 & 0.66 & 0.32 & 0.44 & 0.84 \\
\hline & 0.4 & 6.13 & 3.34 & 1.84 & 0.29 & 0.67 & 0.33 & 0.45 & 0.85 \\
\hline & 0.6 & 6.15 & 3.35 & 1.84 & 0.29 & 0.67 & 0.33 & 0.45 & 0.85 \\
\hline & 0.8 & 6.17 & 3.36 & 1.84 & 0.29 & 0.68 & 0.33 & 0.46 & 0.86 \\
\hline & 1.0 & 6.18 & 3.37 & 1.83 & 0.29 & 0.68 & 0.33 & 0.46 & 0.86 \\
\hline & 2.0 & 6.23 & 3.39 & 1.84 & 0.29 & 0.69 & 0.34 & 0.47 & 0.87 \\
\hline & 4.0 & 6.29 & 3.42 & 1.84 & 0.29 & 0.71 & 0.34 & 0.48 & 0.89 \\
\hline & 6.0 & 6.34 & 3.45 & 1.84 & 0.29 & 0.72 & 0.35 & 0.48 & 0.91 \\
\hline \multirow{8}{*}{$\begin{array}{l}485 \mathrm{~A} \cdot 39-4,47-55 \\
\varrho=2.92 \mathrm{~g} / \mathrm{cm}^{3} \\
\phi=0.6 \%\end{array}$} & 0.2 & 6.18 & 3.32 & 1.86 & 0.30 & 0.69 & 0.32 & 0.47 & 0.84 \\
\hline & 0.4 & 6.23 & 3.34 & 1.87 & 0.30 & 0.70 & 0.33 & 0.48 & 0.85 \\
\hline & 0.6 & 6.27 & 3.35 & 1.87 & 0.30 & 0.71 & 0.33 & 0.49 & 0.85 \\
\hline & 0.8 & 6.30 & 3.36 & 1.88 & 0.30 & 0.71 & 0.33 & 0.50 & 0.86 \\
\hline & 1.0 & 6.33 & 3.37 & 1.88 & 0.30 & 0.73 & 0.33 & 0.51 & 0.86 \\
\hline & 2.0 & 6.40 & 3.40 & 1.88 & 0.30 & 0.75 & 0.34 & 0.52 & 0.88 \\
\hline & 4.0 & 6.45 & 3.43 & 1.88 & 0.30 & 0.76 & 0.34 & 0.53 & 0.90 \\
\hline & 6.0 & 6.48 & 3.46 & 1.87 & 0.30 & 0.76 & 0.35 & 0.53 & 0.91 \\
\hline
\end{tabular}

Table 2. Estimated modal analyses for Leg 65 basalts. $^{\mathrm{a}}$

\begin{tabular}{|c|c|c|c|c|c|c|}
\hline $\begin{array}{c}\text { Sample } \\
\text { (interval in } \mathrm{cm} \text { ) }\end{array}$ & $\begin{array}{l}\text { Plagio- } \\
\text { clase }\end{array}$ & Augite & $\begin{array}{c}\text { Olivine } \\
\text { (altered) }\end{array}$ & Opaques & $\begin{array}{l}\text { Ground- } \\
\text { mass }\end{array}$ & $\begin{array}{l}\text { Filled } \\
\text { Vugs }\end{array}$ \\
\hline 482B-16-2, 107-123 & 52 & 33 & 2 & 3 & 10 & - \\
\hline $482 \mathrm{C}-10-3,73-85$ & 51 & 33 & 4 & 5 & 7 & - \\
\hline $482 C-13-2,117-128$ & 48 & 37 & 5 & 7 & 3 & - \\
\hline $482 \mathrm{C}-14-2,55-65$ & 40 & 40 & 5 & 8 & 7 & - \\
\hline 482D-9-1, 69-81 & 40 & 40 & 6 & 12 & - & 2 \\
\hline 482D-11-3, 0-8 & 28 & 52 & 6 & 12 & - & 2 \\
\hline $483-15-2,129-140$ & 52 & 35 & 6 & 4 & 2 & 1 \\
\hline $483-17-2,130-145$ & 46 & 41 & 8 & 2 & 3 & - \\
\hline $483-20-1,69-81$ & 30 & 48 & 4 & 6 & 12 & - \\
\hline $483-22-3,77-89$ & 28 & 43 & 3 & 8 & 14 & 4 \\
\hline $483-24-1,59-66$ & 37 & 38 & 3 & 7 & 15 & - \\
\hline $483-26-2,133-139$ & 36 & 38 & 4 & 8 & 11 & 3 \\
\hline $483 \mathrm{~B}-4-2,55-65$ & 47 & 39 & 5 & 3 & 5 & 1 \\
\hline $483 \mathrm{~B}-4-4,139-149$ & 55 & 33 & 3 & 4 & 4 & 1 \\
\hline 483B-7-2, 104-114 & 44 & 40 & 5 & 6 & 3 & - \\
\hline $483 \mathrm{~B}-12-2,0-11$ & 42 & 35 & 4 & 6 & 11 & 2 \\
\hline $483 \mathrm{~B}-14-1,102-111$ & 38 & 40 & 2 & 6 & 14 & \\
\hline 483B-19-3, $62-70$ & 43 & 30 & 6 & 4 & 15 & 2 \\
\hline $483 \mathrm{~B}-21-2,106-116$ & 39 & 41 & 4 & 14 & - & 2 \\
\hline 483B-23-2, 97-107 & 43 & 41 & 2 & 5 & 6 & 3 \\
\hline 483B-25-2, $143-150$ & 38 & 46 & - & 7 & 8 & 1 \\
\hline $483 \mathrm{~B}-27-3,76-83$ & 45 & 37 & 2 & 3 & 13 & - \\
\hline $483 \mathrm{~B}-31-2,19-29$ & 33 & 44 & 2 & 9 & 8 & 3 \\
\hline $485 \mathrm{~A}-12-1,107-114$ & 20 & 25 & - & - & 45 & - \\
\hline $485 \mathrm{~A}-17-1,100-117$ & 40 & 39 & 1 & 8 & 12 & - \\
\hline $485 \mathrm{~A}-23-4,16-25$ & 46 & 40 & 1 & 7 & 6 & - \\
\hline $485 \mathrm{~A}-25-3,33-45$ & 44 & 35 & 6 & 6 & 9 & - \\
\hline $485 \mathrm{~A}-29-3,38-48$ & 49 & 39 & 4 & 5 & 3 & - \\
\hline $485 \mathrm{~A}-31-2,94-101$ & 59 & 25 & - & 6 & 10 & - \\
\hline $485 \mathrm{~A}-33-1,32-42$ & 47 & 30 & 1 & 7 & 14 & - \\
\hline $485 \mathrm{~A}-35-1$ & 48 & 26 & 4 & 6 & 16 & - \\
\hline $485 \mathrm{~A}-39-4,47-55$ & 45 & 22 & 13 & 4 & 15 & - \\
\hline
\end{tabular}

${ }^{a}$ Estimated modal analyses in vol. \% 


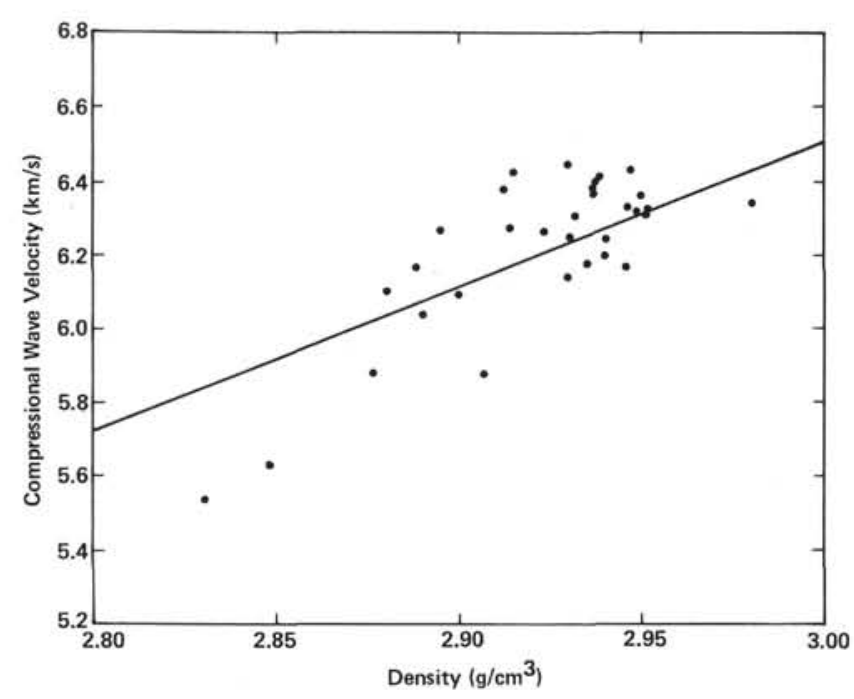

Figure 2. Density vs. compressional wave velocity for Leg 65 basalts at $1.0 \mathrm{kbar}$ confining pressure. (The solid line represents the leastsquares fit to DSDP velocity data summarized by Christensen and Salisbury, 1975.)

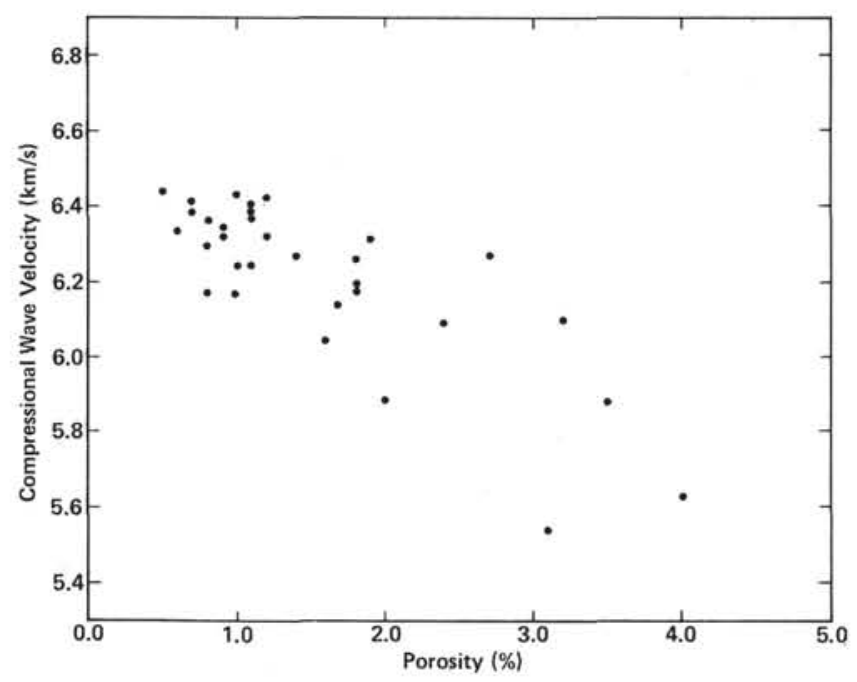

Figure 3. Compressional wave velocity at $1.0 \mathrm{kbar}$ confining pressure vs. porosity for Leg 65 basalts.

The least-squares linear velocity-density solution $V_{p}=$ $3.52 \mathrm{e}-4.10$, previously determined for DSDP basalts by Christensen and Salisbury (1975), is also shown in Fig. 2. Although small, the range of velocities observed correlates well with porosity. This is illustrated in Figure 3 , where compressional wave velocities at $1 \mathrm{kbar}$ confining pressure are plotted vs. measured porosities. The relationship between bulk density and porosity is shown in Figure 4.

A comparison of the velocity model in Figure 1 with the velocity-porosity data in Figure 3 can be used to make several interesting inferences. First, if the extremal bounds from the travel time inversion are used,

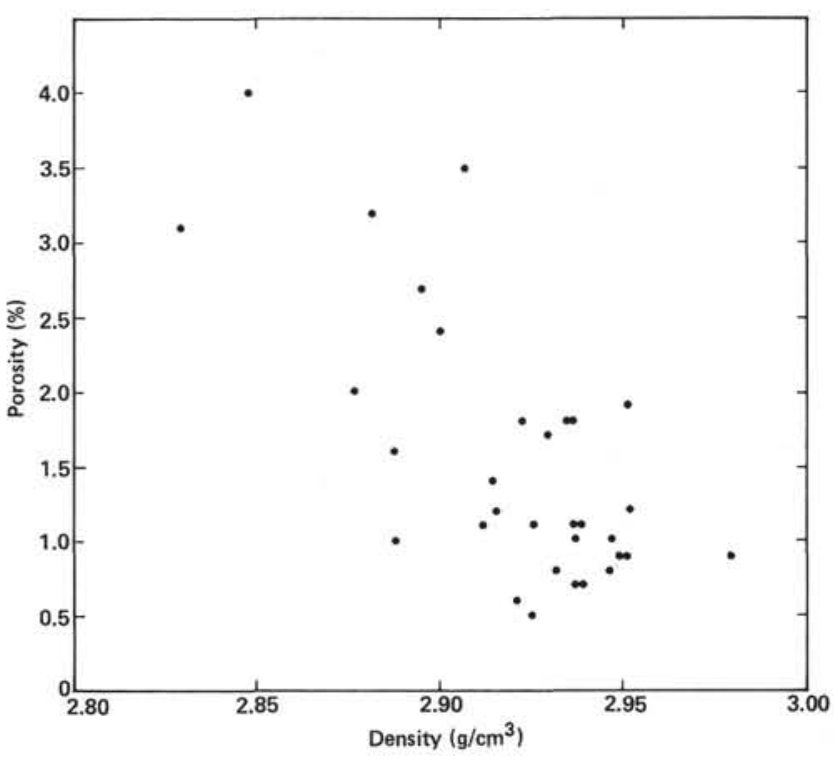

Figure 4. Porosity vs. bulk density for Leg 65 basalts.

the porosity of the top few hundred meters of basalt is constrained to between a few percent and about 10 percent (extrapolating the velocity-porosity results to 4.5 $\mathrm{km} / \mathrm{s}$ ). The preferred velocity model is that derived from synthetic seismograms, and this would favor the higher porosity values. Second, the velocity model from the synthetic seismograms exceeds the maximum measured basalt velocity at a depth of about $2 \mathrm{~km}$ below the seafloor, implying that below this depth the crust cannot be composed of basalt, in agreement with earlier conclusions by Christensen and Salisbury (1975). Since the refraction data do not indicate any first-order discontinuities in the upper crust, it is suggested that the velocity model can be explained by a combination of decreasing porosity and changing petrology with depth.

\section{ACKNOWLEDGMENTS}

This research was supported by the Office of Naval Research Contract N-00014-75-C-0502. The help of S. Lundquist, S. Pollock, L. Slater, and R. Wilkens in the laboratory measurements is greatly appreciated.

\section{REFERENCES}

Birch, F, 1960. The velocity of compressional waves in rocks to 10 kbar. 1. J. Geophys. Res., 65:1083-1102.

1961. The velocity of compressional waves in rocks to 10 kbar. 2. J. Geophys. Res., 65:2199-2224, 1961.

Christensen, N. I., and Salisbury, M. H., 1975. Structure and constitution of the lower oceanic crust. Rev. Geophys. and Space Phys., 13:57-86.

Christensen, N. I., and Shaw, G. H., 1970. Elasticity of mafic rocks from the Mid-Atlantic Ridge. Geophys. J., 20:271-284.

Lewis, B. T. R., 1979. Periodicities in volcanism and longitudinal magma flow on the East Pacific Rise at $23^{\circ} \mathrm{N}$. Geophys. Res. Lett., 6:753-756.

McClain, J. S., and Lewis, B. T. R., 1980. A seismic experiment at the axis of the East Pacific Rise. Mar. Geol., 35:147-169. 